\title{
Inefficient Magnetic-Field Amplification in Supersonic Laser-Plasma Turbulence
}

\author{
A. F. A. Bott๑, ${ }^{1,2,{ }^{*}}$ L. Chen, ${ }^{1}$ G. Boutoux,${ }^{3}$ T. Caillaud, ${ }^{3}$ A. Duval, ${ }^{3}$ M. Koenig, ${ }^{4,5}$ B. Khiar, ${ }^{6}$ I. Lantuéjoul, ${ }^{3}$ \\ L. Le-Deroff $\odot,{ }^{7}$ B. Reville $\odot,{ }^{8}$ R. Rosch, ${ }^{3}$ D. Ryu $\odot,{ }^{9}$ C. Spindloe, ${ }^{10}$ B. Vauzour, ${ }^{3}$ B. Villette, ${ }^{3}$ \\ A. A. Schekochihin $\odot,{ }^{1,11}$ D. Q. Lamb, ${ }^{6}$ P. Tzeferacos, ${ }^{1,6,12,13}$ G. Gregori, ${ }^{1}$ and A. Casner $\odot^{3,14}$ \\ ${ }^{1}$ Department of Physics, University of Oxford, Parks Road, Oxford OX1 3PU, United Kingdom

(Received 20 August 2020; revised 7 June 2021; accepted 17 September 2021; published 21 October 2021)

\begin{abstract}
We report a laser-plasma experiment that was carried out at the LMJ-PETAL facility and realized the first magnetized, turbulent, supersonic $\left(\mathrm{Ma}_{\mathrm{turb}} \approx 2.5\right)$ plasma with a large magnetic Reynolds number $(\mathrm{Rm} \approx 45)$ in the laboratory. Initial seed magnetic fields were amplified, but only moderately so, and did not become dynamically significant. A notable absence of magnetic energy at scales smaller than the outer scale of the turbulent cascade was also observed. Our results support the notion that moderately supersonic, low-magnetic-Prandtl-number plasma turbulence is inefficient at amplifying magnetic fields compared to its subsonic, incompressible counterpart.
\end{abstract}

DOI: 10.1103/PhysRevLett.127.175002

Understanding the evolution of magnetic fields in supersonic plasma turbulence is a challenge that has an intrinsic interest in fluid dynamics and plasma physics, as well as important applications in astrophysics and space science. The intrinsic interest derives from the wide range of physical processes that can arise in magnetized, supersonic plasma turbulence, promising an exceptionally rich collection of complex phenomena for study. As for the applications, magnetic fields are believed to play a significant role in the turbulent, supersonic dynamics of the interstellar medium (ISM); understanding the complex interactions between the fields, shocks and vortices present in such an environment is a necessary component of a comprehensive picture of the ISM, encompassing important topics such as star formation [1-4]. Magnetized,

Published by the American Physical Society under the terms of the Creative Commons Attribution 4.0 International license. Further distribution of this work must maintain attribution to the author(s) and the published article's title, journal citation, and DOI. moderately supersonic plasma turbulence is also thought to emerge in solar and stellar convection zones [5,6].

One key question concerning the relationship between magnetic fields and supersonic plasma turbulence is the conditions under which the fields attain dynamical strengths (as, for example, observed in the ISM). The equivalent question in subsonic plasma turbulence has been studied in depth, mostly within the framework of magnetohydrodynamics (MHD). Analytical theory [7-11], simulations [12-16], liquid-metal experiments [17-19], and laser-plasma experiments [20-23] give a consistent picture, showing that chaotic bulk motions of magnetized fluid (with characteristic scale $L$ and velocity $u_{\text {rms }}$ ) can amplify any small seed magnetic field initially present in that fluid provided the magnetic Reynolds number $\mathrm{Rm} \equiv$ $u_{\mathrm{rms}} L / \eta$ is greater than a certain critical value $\mathrm{Rm}_{c}$ (here, $\eta$ is the plasma resistivity). This critical value is usually significantly larger than unity [24]. For $1 \ll \mathrm{Rm} \lesssim \mathrm{Rm}_{c}$, the magnitude $\delta B$ of the magnetic field postamplification is related to the magnitude $B_{0}$ of the initial seed field via $\delta B \sim \mathrm{Rm}^{1 / 2} B_{0}$ [15]. However, if $\mathrm{Rm}>\mathrm{Rm}_{c}$, magneticfield amplification of seed fields proceeds unabated until 
the magnetic-energy density of the amplified field reaches equipartition with the kinetic-energy density of the stochastic motions responsible for the amplification; this fieldamplification mechanism is known as the fluctuation dynamo. Another important parameter for magnetic-field amplification is the magnetic Prandtl number $\mathrm{Pm} \equiv$ $\mathrm{Rm} / \mathrm{Re}$ (where Re is the fluid Reynolds number): dynamo is generally less efficient for $\mathrm{Pm} \ll 1$ than for $\mathrm{Pm} \gtrsim 1$ (e.g., $\mathrm{Rm}_{c}$ increases as Pm decreases [10,15,25-27]).

There exist far fewer theoretical and numerical studies of magnetic-field amplification in supersonic plasma turbulence as compared with the subsonic case, and no experimental observations in the supersonic regime. The latter is an unfortunate omission, because numerical studies of supersonic MHD turbulence [28-31] indicate that, while the fluctuation dynamo is still capable of operating, the efficacy of the mechanism, both in terms of the characteristic growth rates of magnetic fields and saturated magnetic to kinetic energy ratios, is lessened when the turbulent Mach number $\mathrm{Ma}_{\text {turb }} \equiv u_{\mathrm{rms}} / c_{s}$ (where $c_{s}$ is the plasma's sound speed) is increased to order unity. Physically, this has been attributed to various factors: reduced energy available to the solenoidal stretching motions necessary for dynamo action on account of some of the driving kinetic energy flux being directed towards compressive motions, irrespective of the driving mechanism [28,29,32-34]; a steepened turbulent velocity spectrum [35]; and enhanced dissipation of magnetic fields in shocks [36]. Laboratory investigations of the supersonic regime that test these expectations have been limited by the impossibility of its realization in liquid-metal dynamo experiments, which are all in the subsonic regime [37]. As for laser-plasma experiments, there has only been one previous experiment that successfully realized boundary-free, supersonic plasma turbulence [38]; however, $\mathrm{Rm}$ achieved in that experiment was much smaller than unity, prohibiting significant magnetic-field amplification.

In this Letter, we report a new laser-plasma experiment that managed to create supersonic, high-Rm plasma turbulence for the first time in the laboratory. The experiment was performed on the Laser Megajoule (LMJ) facility in Bordeaux [39]. The platform employed for the experiment is depicted in Fig. 1. Similarly to previous laser-plasma experiments investigating the fluctuation dynamo in subsonic plasma [22,23], a turbulent plasma was created by colliding inhomogeneous, asymmetric, counterpropagating rear-side-blow-off plasma jets. Spatial inhomogeneity is introduced by placing grids in the paths of each jet prior to their collision; the jet asymmetry follows directly from using asymmetric grids. In order to reach the supersonic regime, several major design modifications to a previous experiment [23] were introduced. The thickness of the foils irradiated by the LMJ drive beams was reduced, and the beam energy per foil increased fourfold: both changes led to increased initial jet velocities. In addition, aluminium rather than plastic foils were used in the experiment; the

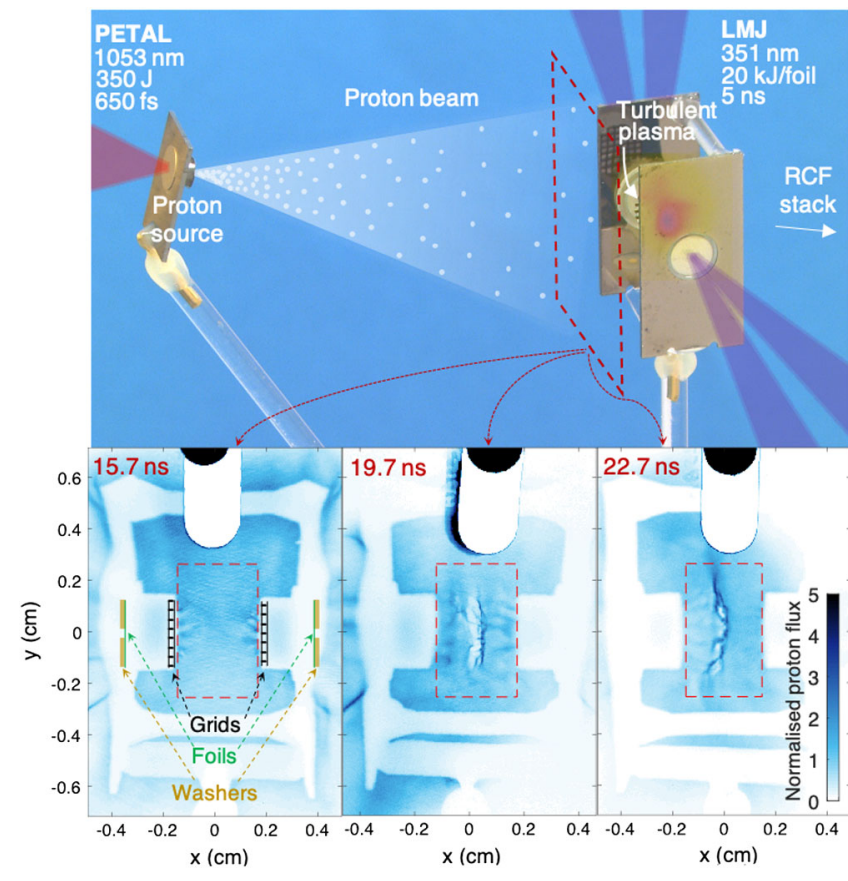

FIG. 1. Experimental setup. Upper panel: annotated photograph of one of the targets used in our experiment. The main target is rendered partially transparent, in order to show the location of the turbulent plasma (the yellow-purple region). The targets' technical specifications are given in [42]. Lower panels: $8.5 \mathrm{MeV}$ proton images (obtained from different experimental shots) at $15.7 \mathrm{~ns}$ (left), $19.7 \mathrm{~ns}$ (middle) and $22.7 \mathrm{~ns}$ (right) after the initiation of the drive beams. The proton flux normalization is defined relative to the mean of the regions enclosed by red-dashed lines in each image.

resulting enhancement in radiative cooling reduced the plasma's temperature both before and after jet collision. Both modifications were anticipated to increase $\mathrm{Ma}_{\text {turb }}$, a claim supported by three-dimensional, three-temperature radiation-MHD simulations performed concurrently to the experiment using the FLASH code $[40,41]$.

The primary diagnostic on the experiment, CRACC (Cassette de Radiographie au Centre Chambre) [55], provides time-resolved proton imaging [56], which was used to measure magnetic fields and the electron number density in the plasma, as well as to determine the characteristic velocities of the initial jets. The proton imaging beam was generated by irradiating a gold foil with the highintensity PETAL beam (see Fig. 1) [57]; via the target normal sheath acceleration (TNSA) mechanism [58], this irradiation results in a highly directed proton beam with a thermal ( $\sim 3 \mathrm{MeV}$ temperature) spectrum. The beam passed through the plasma generated by the LMJ drive beams, and subsequently was detected using a calibrated radiochromic film $(\mathrm{RCF})$ stack $[42,55]$. The RCF stack was designed in such a way that protons with distinct energies were detected in separate layers of RCF ( $\sim 0.5 \mathrm{MeV}$ energy resolution); this allowed for time-resolved measurements on each 
experimental shot, because slower beam protons passed through the plasma at later times than faster ones. The $\Delta t_{p} \approx 300 \mathrm{ps}$ time delay between the fastest and slowest detected protons (8.5 MeV vs $4.7 \mathrm{MeV}$ ) was too small to capture the full dynamical evolution of the plasma turbulence; to capture this evolution, we repeated our experiment, but with three different relative offsets between the LMJ and PETAL beams. The resulting proton images (for the $8.5 \mathrm{MeV}$ protons) are shown in Fig. 1.

Detailed quantitative information about the magnetic fields present in the turbulent plasma can be obtained by analyzing the proton images. The theoretical basis for such analysis comes from the proton beam's high velocity and low density compared to that of the plasma with which it interacts prior to reaching the RCF stack; inhomogeneities in the detected proton flux can therefore be attributed to the action on the beam protons of the Lorentz forces arising from spatially varying magnetic fields present in the plasma [59]. This being the case, recent work [60] has shown that the two components of the path-integrated magnetic field that are perpendicular to the proton beam's direction can be reconstructed directly from these inhomogeneities, provided the proton beam, on account of its nonuniform distortion, does not self-intersect before reaching the detector. Further technical details about this analysis are given in [42].

The velocity $u_{\text {jet }}$ of the supersonic plasma jets prior to their collision can be determined from the time evolution of the path-integrated magnetic field that we reconstructed from the $8.5 \mathrm{MeV}$ proton image of the jets (given in Fig. 2, top left). The dominant component of the path-integrated field (characteristic magnitude $\sim 0.4 \mathrm{kG} \mathrm{cm}$ ) inside the main bulk of each jet is aligned with the jets' predominant direction of motion, and oscillates strongly in the direction normal to it. To extract the velocity of the left-hand jet, we compare the path-integrated field recovered from 4.7 and $8.5 \mathrm{MeV}$ proton images generated on the same experimental shot (see Fig. 2, top right), corresponding to imaging times of 15.7 and $16.0 \mathrm{~ns}$, respectively. While the morphology of both images is very similar, the characteristic extent in the parallel direction of the oscillatory pathintegrated field structure is slightly greater at $16.0 \mathrm{~ns}$ (see Fig. 2, bottom left). We attribute this finding to the motion of the magnetic fields inside the jet: these fields are frozen into the bulk flow provided the jet's magnetic Reynolds number $\mathrm{Rm}_{\text {jet }} \equiv u_{\mathrm{jet}} L / \eta$ (where $L=0.04 \mathrm{~cm}$ is the grid periodicity) just after its interaction with the grid satisfies $\mathrm{Rm}_{\text {jet }} \gg 1$ (an assumption supported by theoretical expectations concerning the initial jet properties [42]). The mean jet velocity $u_{\text {jet }}$ is obtained as follows: we calculate average lineouts for five different regions of width $L$ (depicted in Fig. 2, top right) for the path-integrated fields measured at each time (two sample lineouts are shown in Fig. 2, bottom right); then we determine the mean spatial offset $\Delta x_{p}$ between each temporal pair of lineouts; finally we estimate
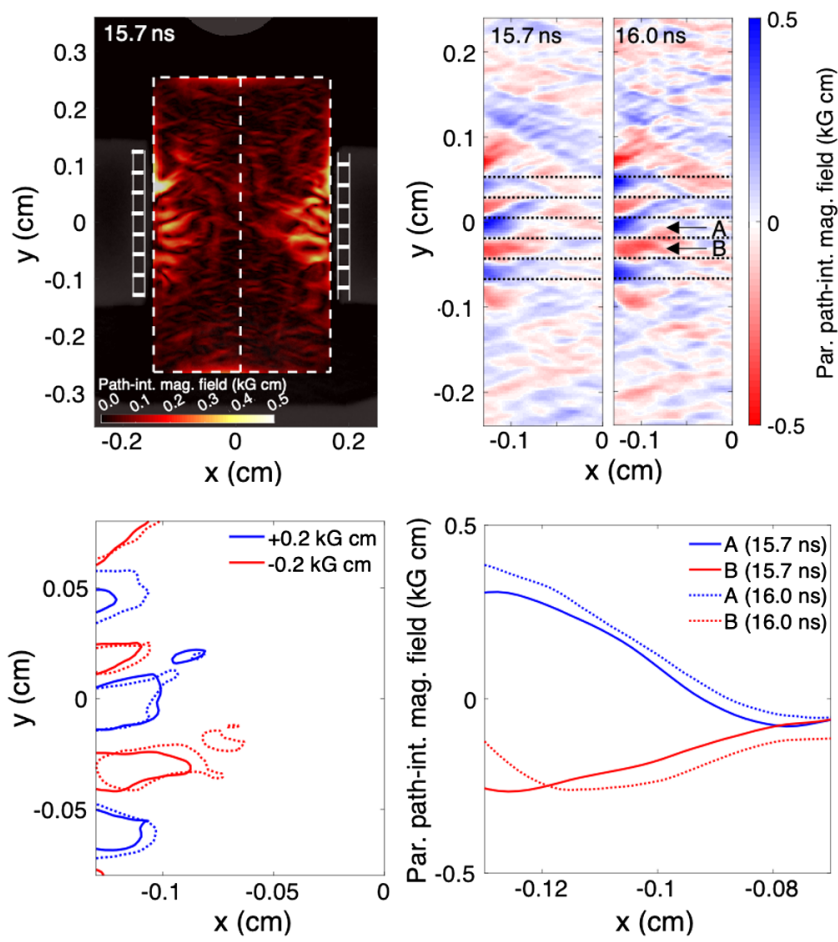

FIG. 2. Jet-velocity measurement. Top left: magnitude of perpendicular path-integrated magnetic field reconstructed from the region denoted in the 15.7 ns proton image shown in Fig. 1. The procedure used to extract this quantity is described in [42] (see also [60]). Top right: axial component of the path-integrated magnetic field determined at $15.7 \mathrm{~ns}$ and $16.0 \mathrm{~ns}$ by analyzing 8.5 MeV and 4.7 MeV proton images, respectively. Bottom left: $\pm 0.2 \mathrm{kG} \mathrm{cm}$ contour plots of the axial path-integrated magnetic field components at $15.7 \mathrm{~ns}$ (solid) and $16.0 \mathrm{~ns}$ (dotted). Bottom right: lineouts of axial path-integrated magnetic-field component, calculated from the regions " $A$ " and " $B$ " shown above.

$u_{\text {jet }}$ via $u_{\text {jet }} \approx \Delta x_{p} / \Delta t_{p}$. We find $u_{\text {jet }}=290 \pm 40 \mathrm{~km} / \mathrm{s}$, a value that is consistent with heuristic estimates [42].

Once collision between the jets has occurred, x-ray imaging data from related experiments on other laser facilities [22,23] indicates that a turbulent plasma with higher characteristic temperatures and densities quickly coalesces. The burst of self-emitted $\mathrm{x}$ rays that coincides with this coalescence can be used to measure the turbulent plasma's temperature, $T$. The spectrum of these $\mathrm{x}$ rays was measured in our experiment using the DMX diagnostic $[61,62]$; DMX is an absolutely calibrated, time-resolved broadband spectrometer with high temporal resolution ( $\simeq 100 \mathrm{ps})$. The brightness temperature of the 10 lower energy channels allow for $T$ to be extracted: $T=100 \pm$ $30 \mathrm{eV}$ (with the uncertainty mostly coming from the uncertainty in the emitting surface area of the plasma).

The characteristic electron number density $n_{e}$ of the interaction-region plasma can be determined concurrently by quantifying the effect of collisional scattering on the resolution of the sharp, large-amplitude proton-flux inhomogeneities ("caustics") present in the $4.7 \mathrm{MeV}$ proton 
images [42]. We find $n_{e}=(4 \pm 1) \times 10^{19} \mathrm{~cm}^{-3}$ at $t=19.7 \mathrm{~ns}$ and $n_{e}=(7 \pm 2) \times 10^{19} \mathrm{~cm}^{-3}$ at $t=22.7 \mathrm{~ns}$, values which are consistent with measurements from related experiments [22,23].

Given our measurement of $u_{\text {jet }}$, the characteristic turbulent velocity $u_{\text {turb }}$ in the interaction-region plasma can be estimated as follows. X-ray measurements from previous experiments [23] and FLASH simulations [41] indicate that, while the jet velocities are close to being uniform transversely, the density of either of the plasma flows is much larger at transverse spatial positions coincident with the locations of the grid holes through which that flow has passed than the density at the analogous position in the opposing flow. When the two plasma flows collide, conservation of momentum therefore dictates that the flow velocity in these transverse spatial locations will be close to the higher-density plasma flow's incident velocity. Taking into consideration the two-dimensional periodic reversals in the flow direction, and assuming that this flow profile is efficiently randomized by nonlinear interactions and/or Kelvin-Helmholtz instabilities, we conclude that $u_{\text {turb }} \approx u_{\text {jet }} / \sqrt{2}=200 \pm 30 \mathrm{~km} / \mathrm{s}$.

The sound speed in the plasma is $c_{s}=\sqrt{\gamma(Z+1) T / m_{i}} \approx$ $80 \pm 10 \mathrm{~km} / \mathrm{s}$, where $\gamma$ is the adiabatic index, $Z$ the plasma's ionization state, and $m_{i}$ the ion mass. Therefore, the turbulent Mach number is $\mathrm{Ma}_{\text {turb }}=2.5 \pm 0.5$, so the turbulence is supersonic.

Using all this information, the plasma's viscosity and resistivity-and thus the fluid and magnetic Reynolds numbers-are determined via known expressions for transport coefficients in a collisional, aluminium plasma [42]. We find that $\operatorname{Re}=(1.2 \pm 1.0) \times 10^{6}$, a Reynolds number which (irrespective of the significant uncertainty on its exact value) is certainly large enough to allow for the formation of a developed turbulent cascade. The magnetic Reynolds number is also significantly larger than unity, but is much smaller than Re: $\mathrm{Rm}=45 \pm 20$, so $\mathrm{Pm} \sim 10^{-5}-10^{-4}$. Much smaller values of $\mathrm{Pm}$ are obtained in this experiment compared with previous experiments that created turbulent laser plasmas with similar kinetic-energy densities [22,23] because of the distinct material composition of the plasma; the comparatively higher charge of aluminum ions, as well as the lower temperatures arising from enhanced radiative cooling of an aluminum plasma, both act to reduce Pm, which scales as $\mathrm{Pm} \propto Z^{-4} T^{4}$ at fixed $n_{e}$ [42].

The seed magnetic fields initially present in the interaction-region plasma, and the stochastic field structures arising from the interaction of those seed fields with the supersonic plasma turbulence, can both be characterized using the path-integrated magnetic-field maps extracted from $8.5 \mathrm{MeV}$ proton images after the jet collision. The seed fields, which are generated at the laser spots by the Biermann battery [63] and subsequently advected into
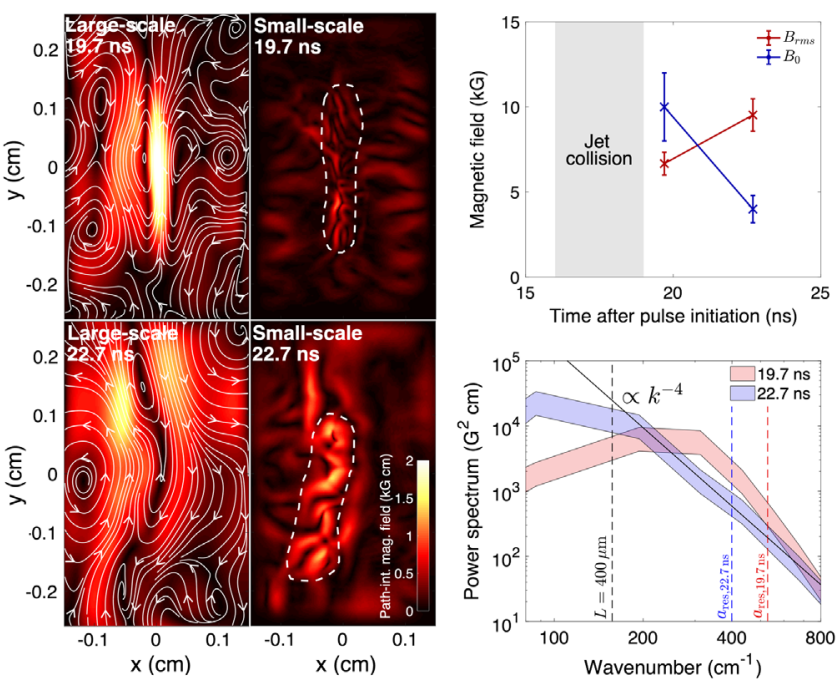

FIG. 3. Magnetic-field measurements. Left: magnitude of perpendicular path-integrated seed (large-scale) magnetic fields at $19.7 \mathrm{~ns}$ (top) and $22.7 \mathrm{~ns}$ (bottom). The streamlines of this field are also depicted. Middle: magnitude of perpendicular pathintegrated stochastic magnetic fields. Top right: evolution of seed (blue) and stochastic (red) magnetic field over time. Bottom right: magnetic-energy spectra calculated in demarcated regions from maps of path-integrated stochastic field components. The anticipated resolution limits on our spectra imposed by collisional scattering of the $8.5-\mathrm{MeV}$ proton beam are also shown.

the interaction region, have a characteristic transverse scale comparable to that of the interaction region $\left(\ell_{n \perp} \approx 0.25 \mathrm{~cm}\right)$, while the correlation length of the stochastic fields is significantly smaller $\left(\ell_{B} \approx 150 \mu \mathrm{m}\right)$. We take advantage of this scale separation to extract distinct path-integrated field maps for the seed and stochastic magnetic fields in the experiment (see Fig. 3). The extraction procedure is explained in [42].

We estimate the characteristic magnitude $B_{0}$ of the seed magnetic fields via a simple relation [59]: $B_{0} \approx$ $10\left[B_{\text {path }, 0}(\mathrm{kG} \mathrm{cm}) / 2 \mathrm{kG} \mathrm{cm}\right]\left[\ell_{n \perp}(\mathrm{cm}) / 0.25 \mathrm{~cm}\right]^{-1} \mathrm{kG}$, where $B_{\text {path, } 0}$ is the characteristic magnitude of the pathintegrated seed magnetic field. The field magnitude obtained just after the collision has occurred $\left(B_{0} \approx 10 \mathrm{kG}\right.$ at $t=19.7 \mathrm{~ns}$ after the LMJ drive beams are initiated) is consistent with related experiments [23]. At $t=22.7 \mathrm{ns,}$ which is over one driving-scale eddy turnover time $\tau_{L} \equiv$ $L / u_{\text {rms }}$ after the collision $\left(\tau_{L} \approx 2 \mathrm{~ns}\right)$, the seed magnetic fields decay $\left(B_{0} \approx 4 \mathrm{kG}\right)$, which can be attributed to their dilution by the interaction-region plasma's expansion, and turbulent diffusion.

The stochastic component of the magnetic field is characterized by its energy spectrum $E_{B}(k)$, which describes the distribution of the magnetic energy amongst different length scales. We determine $E_{B}(k)$ from the path-integrated map of the stochastic magnetic field by assuming statistical homogeneity and isotropy; under these assumptions, it can 
be shown that $E_{B}(k)=k E_{\text {path }}(k) / 4 \pi^{2} \ell_{n \perp}$, where $E_{\text {path }}(k)$ is the one-dimensional spectrum of the path-integrated field [60]. The root mean square of the stochastic magnetic field, $\delta B_{\text {rms }}$, can then be calculated directly from $E_{B}(k)$ as $\delta B_{\mathrm{rms}}=\left[8 \pi \int_{0}^{\infty} d k E_{B}(k)\right]^{1 / 2}$. We find that at $t=19.7 \mathrm{~ns}$, $\delta B_{\text {rms }} \approx 6 \mathrm{kG}$, before subsequently attaining magnitudes comparable in strength to the initial seed fields $\left(\delta B_{\mathrm{rms}} \approx 10 \mathrm{kG}\right.$ at $t=22.7 \mathrm{~ns}$-see Fig. 3). The magneticenergy spectra at both times have steep power-law tails $E_{B}(k) \propto k^{-4}$, with the spectral peaks at wave number $k_{\text {peak }} \approx 2 \pi / L$.

Our measurements suggest that amplification of the magnetic fields by the supersonic, low-Pm turbulence is quite inefficient, in spite of $\mathrm{Rm}$ being significantly greater than unity. This inefficiency is most directly revealed by comparing the peak amplification factor of the seed magnetic field ( $\sim 2.5$ at $t=22 \mathrm{~ns})$ with equivalent factors obtained in previous subsonic laser-plasma turbulence experiments; the degree of amplification is similar to that seen in experiments with similar Pm, but much lower $\mathrm{Rm} \sim 3-7$ [21], while it is an order of magnitude smaller than in other experiments with both larger values of $\mathrm{Rm}$ and $\mathrm{Pm}[22,23,42]$. The first comparison suggests that the efficiency of magnetic-field amplification is reduced as $\mathrm{Ma}_{\text {turb }}$ is increased above unity, while the second suggests the efficiency is independently improved as Rm and Pm are increased. Both findings are consistent with expectations based on periodic-box simulations of compressible turbulent flows that show how dynamo efficiency varies with $\mathrm{Ma}_{\text {turb }}, \mathrm{Rm}$, and $\mathrm{Pm}[29,30]$. The degree of amplification is also smaller than that attained in liquid-metal experiments with similar MHD parameters (see, e.g., [18] in which $B_{\text {rms }} / B_{0} \gtrsim 30$ at $\mathrm{Rm} \approx 45$ ), although a direct comparison is challenging to make due to the differing flow geometries.

Another metric by which the inefficiency of magneticfield amplification can be assessed in the experiment is the magnetic-kinetic energy ratio, which is $E_{\mathrm{mag}} / E_{\mathrm{kin}} \approx 10^{-4}$ at $t=22 \mathrm{~ns}$. This value is well below those found in subsonic dynamo experiments postamplification at larger $\mathrm{Rm}$ and $\mathrm{Pm}[22,23,42]$, or the saturation values obtained in simulations of MHD turbulent supersonic dynamos with $\mathrm{Ma}_{\text {turb }}=2.4$ and order-unity Pm [29]. These comparisons corroborate the amplifications inefficiency; they could further be interpreted to suggest that we did not reach the bona fide dynamo regime in our experiment, in turn providing a lower bound $\mathrm{Rm}_{c}>45$ on $\mathrm{Rm}_{c}$ for $\mathrm{Pm} \ll 1$ plasma turbulence with $\mathrm{Ma}_{\text {turb }} \gtrsim 1$. By comparison, dynamo action has been observed in MHD simulations with $\mathrm{Ma}_{\text {turb }}=2.6$ and $\mathrm{Pm}=1$ at $\mathrm{Rm} \gtrsim 80$ [28], and in some (subsonic) $\mathrm{Pm} \ll 1$ liquid-metal experiments with $\mathrm{Rm} \gtrsim 30$ [18].

In summary, our experiment has demonstrated magneticfield amplification in a supersonic, turbulent plasma for the first time in the laboratory, and our results support the theoretical expectation that magnetic-field amplification is less efficient in supersonic turbulence compared to subsonic turbulence. Looking forward, future laboratory investigations of amplification in supersonic vs subsonic laser-plasma flows with similar values of $\mathrm{Rm}$ and $\mathrm{Pm}$ will be possible with only minor modifications to our current platform. Furthermore, we believe that creating a more efficient laser-plasma turbulent dynamo in the supersonic regime in future experiments is feasible. FLASH simulations of our experiment, which (by assuming more efficient laser-target energy coupling than was attained in the experiment) realized characteristic kinetic and thermal energies 3-4 times greater than we report here, achieve $\mathrm{Rm} \approx 750$, and also show rapid magnetic-field amplification [41]. This suggests that exploring the transition to the dynamo regime in the laboratory is possible: an attractive project.

The research leading to these results received funding from the Engineering and Physical Sciences Research Council, Grants No. EP/M022331/1, No. EP/N014472/1, and No. EP/RO34737/1, and the U.S. Department of Energy (DOE) National Nuclear Security Administration (NNSA) under Field Work Proposal No. 57789 to ANL, Subcontract No. 536203 with LANL, Subcontract B632670 with LLNL, and Grants No. DE-NA0002724, No. DE-NA0003605, and No. DE-NA0003934 to the University of Chicago, No. DE-NA0003539 to the Massachusetts Institute of Technology, and Cooperative Agreement DE-NA0003856 to the LLE, University of Rochester. We acknowledge support from the U.S. DOE Office of Science Fusion Energy Sciences under Grant No. DE-SC0016566 and the National Science Foundation under Grants No. PHY-1619573, No. PHY-2033925, and No. AST-1908551, the France And Chicago Collaborating in The Sciences (FACCTS) Program, and Grants No. 2016R1A5A1013277 and No. 2020R1A2C2102800 of the National Research Foundation of Korea. Awards of computer time were provided by the U.S. Department of Energy Innovative and Novel Computational Impact on Theory and Experiment (INCITE) and ASCR Leadership Computing Challenge (ALCC) programs. Simulations supporting this research used resources of the Argonne Leadership Computing Facility at ANL, which is supported by the Office of Science of the U.S. DOE under Contract No. DE-AC02-06CH11357. Support from AWE plc. and the Science and Technology Facilities Council of the United Kingdom is also acknowledged. The PETAL laser was designed and constructed by CEA under the financial auspices of the Conseil Regional d'Aquitaine, the French Ministry of Research, and the European Union. The CRACC diagnostic was designed and commissioned on the LMJ-PETAL facility as a result of the PETAL + project coordinated by University of Bordeaux and funded by the French Agence Nationale de la Recherche under Grant No. ANR-10-EQPX-42-01. The LMJ-PETAL experiment presented in this article was supported by Association Lasers et Plasmas and by CEA. 
We are grateful to the staff of CEA-DAM and LMJ for making the experiment possible. We also thank our three reviewers and Lev Arzamasskiy for their helpful feedback on the manuscript.

*abott@princeton.edu

[1] T. Passot, E. Vazquez-Semadeni, and A. Pouquet, Astrophys. J. 455, 536 (1995).

[2] M.-M. Mac Low and R. S. Klessen, Rev. Mod. Phys. 76, 125 (2004).

[3] M. R. Krumholz and C. Federrath, Front. Astron. Space Sci. 6, 7 (2019).

[4] P. Girichidis, S. S. R. Offner, A. G. Kritsuk, R. S. Klessen, P. Hennebelle, J. M. D. Kruijssen, M. G. H. Krause, S. C. O. Glover, and M. Padovani, Space Sci. Rev. 216, 68 (2020).

[5] F. Cattaneo, N. E. Hurlburt, and J. Toomre, Astrophys. J. Lett. 349, L63 (1990).

[6] A. Nordlund, R. F Stein, and M. Asplund, Living Rev. Solar Phys. 6, 2 (2009).

[7] A. P. Kazantsev, JETP Lett. 26, 1031 (1968), http://jetp.ras .ru/cgi-bin/dn/e_026_05_1031.pdf.

[8] Y. B. Zel'dovich, A. A. Ruzmaikin, S. A. Molchanov, and D. D. Sololov, J. Fluid Mech. 144, 1 (1984).

[9] R. Kulsrud and S. W. Anderson, Astrophys. J. 396, 606 (1992).

[10] S. Boldyrev and F. Cattaneo, Phys. Rev. Lett. 92, 144501 (2004).

[11] A. A. Schekochihin, S. C. Cowley, G. W. Hammett, J. L. Maron, and J. C. McWilliams, New J. Phys. 4, 84 (2002).

[12] M. Meneguzzi, U. Frisch, and A. Pouquet, Phys. Rev. Lett. 47, 1060 (1981).

[13] A. A. Schekochihin, S. C. Cowley, S. F. Taylor, J. L. Maron, and J. C. McWilliams, Astrophys. J. 612, 276 (2004).

[14] N. E. Haugen, A. Brandenburg, and W. Dobler, Phys. Rev. E 70, 016308 (2004).

[15] A. A. Schekochihin, A. B. Iskakov, S. C. Cowley, J. C. McWilliams, M. R. E. Proctor, and T. A. Yousef, New J. Phys. 9, 300 (2007)

[16] A. Seta, P. J. Bushby, A. Shukarov, and T. S. Wood, Phys. Rev. Fluids 5, 043702 (2020).

[17] A. Gailitis, O. Lielausis, E. Platacis, S. Dement'ev, A. Cifersons, G. Gerbeth, T. Gundrum, F. Stefani, M. Christen, and G. Will, Phys. Rev. Lett. 86, 3024 (2001).

[18] R. Monchaux et al., Phys. Rev. Lett. 98, 044502 (2007).

[19] D. S. Zimmerman, S. A. Triana, H.-C. Nataf, and D. P. Lathrop, J. Geophys. Res. Solid Earth 119, 4538 (2014).

[20] J. Meinecke et al., Nat. Phys. 10, 520 (2014).

[21] J. Meinecke et al., Proc. Natl. Acad. Sci. U.S.A. 112, 8211 (2015).

[22] P. Tzeferacos et al., Nat. Commun. 9, 591 (2018).

[23] A. F. A. Bott et al., Proc. Natl. Acad. Sci. U.S.A. 118, e2015729118 (2021).

[24] F Rincon, J. Plasma Phys. 85, 205850401 (2019).

[25] Y. Ponty, P. D. Mininni, D. C. Montgomery, J.-F. Pinton, H. Politano, and A. Pouquet, Phys. Rev. Lett. 94, 164502 (2005).
[26] P. D. Mininni and D. C. Montgomery, Phys. Rev. E 72, 056320 (2005).

[27] S. Kreuzahler, Y. Ponty, N. Plihon, H. Homann, and R. Grauer, Phys. Rev. Lett. 119, 234501 (2017).

[28] N. E. L. Haugen, A. Brandenburg, and A. J. Mee, Mon. Not. R. Astron. Soc. 353, 947 (2004).

[29] C. Federrath, G. Chabrier, J. Schober, R. Banerjee, R. S. Klessen, and D. R. G. Schleicher, Phys. Rev. Lett. 107, 114504 (2011).

[30] C. Federrath, J. Schober, S. Bovino, and D. R. G. Schleicher, Astrophys. J. Lett. 797, L19 (2014).

[31] J. Park and D. Ryu, Astrophys. J. 875, 2 (2019).

[32] D. H. Porter, P. R. Woodward, and A. Pouquet, Phys. Fluids 10, 237 (1998).

[33] S. Boldyrev, A. Nordlund, and P. Padoan, Phys. Rev. Lett. 89, 031102 (2002).

[34] A. J. Mee and A. Brandenburg, Mon. Not. R. Astron. Soc. 370, 415 (2006).

[35] J. Schober, D. Schleicher, S. Bovino, and R. S. Klessen, Phys. Rev. E 86, 066412 (2012).

[36] P. Padoan and A. Nordlund, Astrophys. J. 526, 279 (1999).

[37] G. Verhille, N. Plihon, M. Bourgoin, P. Odier, and J.-F. Pinton, Space Sci. Rev. 152, 543 (2010).

[38] T. G. White et al., Nat. Commun. 10, 1758 (2019).

[39] A. Casner et al., High Energy Density Phys. 17, 2 (2015).

[40] B. Fryxell, K. Olson, P. Ricker, F. X. Timmes, M. Zingale, D. Q. Lamb, P. MacNeice, R. Rosner, J. W. Truran, and H. Tufo, Astrophys. J. 131, S273 (2000).

[41] B. Khiar, P. Tzeferacos, A. F. A. Bott, A. A. Schekochihin, A. Casner, G. Gregori, and D. Q. Lamb (to be published).

[42] See Supplemental Material at http://link.aps.org/ supplemental/10.1103/PhysRevLett.127.175002, which includes Refs. [43-54], for additional detail and analysis.

[43] C. Fauquignon and F. Floux, Phys. Fluids 13, 386 (1970).

[44] D. E. Fratanduono, T. R. Boehly, P. M. Celliers, M. A. Barrios, J. H. Eggert, R. F. Smith, D. G. Hicks, G. W. Collins, and D. D. Meyerhofer, J. Appl. Phys. 110, 073110 (2011).

[45] C. E. Max, Physics of Laser Fusion. Vol. I. Theory of the Coronal Plasma in Laser-Fusion Targets, Report No. UCRL53107, Lawrence Livermore National Laboratory, 1981.

[46] S. Agnostinelli et al., Nucl. Instrum. Methods Phys. Res., Sect. A 506, 250 (2003).

[47] L. Chen et al., Astrophys. J. 892, 114 (2020).

[48] F. Nürnberg et al., Rev. Sci. Instrum. 80, 033301 (2009).

[49] J. D. Huba, NRL Plasma Formulary (Naval Research Laboratory, Washington DC, 1994).

[50] C. M. Huntington et al., Nat. Phys. 11, 173 (2015).

[51] S. I. Braginskii, Rev. Plasma Phys. 1, 205 (1965).

[52] J. E. Cross, B. Reville, and G. Gregori, Astrophys. J. 795, 59 (2014).

[53] J. Abdallah, Jr. and R. E. H. Clark, TOPS: A multigroup opacity code, Los Alamos Report No. LA-10454.

[54] J. Meinecke et al., arXiv:2105.08461.

[55] I. Lantuéjoul et al., Proc. SPIE 10763, 107630X (2018).

[56] A. J. Mackinnon et al., Rev. Sci. Instrum. 75, 3531 (2004). 
[57] N. Blanchot et al., Overview of the 1.15 PW PETAL laser in the LMJ facility in Conference on Lasers and ElectroOptics, OSA Technical Digest (online) (Optical Society of America, 2017), paper SF1K.1, https://doi.org/10.1364/ CLEO_SI.2017.SF1K.1.

[58] S. C. Wilks, A. B. Langdon, T. E. Cowan, M. Roth, M. Singh, S. Hatchett, M. H. Key, D. Pennington, A. MacKinnon, and R. A. Snavely, Phys. Plasmas 8, 542 (2001).

[59] N. L. Kugland, D. D. Ryutov, C. Plechaty, J. S. Ross, and H.-S. Park, Rev. Sci. Instrum. 83, 101301 (2012).
[60] A. F. A. Bott, C. Graziani, T. G. White, P. Tzeferacos, D. Q. Lamb, G. Gregori, and A. A. Schekochihin, J. Plasma Phys. 83, 905830614 (2017).

[61] J. L. Bourgade, B. Villette, J. L. Bocher, J. Y. Boutin, S. Chiche, N. Dague, D. Gontier, J. P. Jadaud, B. Savale, and R. Wrobel, Rev. Sci. Instrum. 72, 1173 (2001).

[62] T. Caillaud et al., Proc. SPIE 9966, 996606 (2016).

[63] G. Gregori, B. Reville, and F. Miniati, Phys. Rep. 601, 1 (2015). 